\begin{tabular}{|c|c|c|}
\hline 18 & $\begin{array}{l}\text { European Association for the } \\
\text { Development of Renewable Energies, Environment } \\
\text { and Power Quality (EA4EPQ) }\end{array}$ & $\begin{array}{l}\text { International Conference on Renewable Energies and Power Quality } \\
\text { (ICREPQ'12) } \\
\text { Santiago de Compostela (Spain), 28th to 30th March, } 2012\end{array}$ \\
\hline
\end{tabular}

\title{
Physico-chemical properties of transformer mineral oils submitted to moisture and electrical discharges
}

\author{
T. Toudja ${ }^{1}$, A. Nacer ${ }^{1}$, H. Moulai ${ }^{1}$, I. Khelfane ${ }^{2}$ and A. Debche ${ }^{3}$ \\ ${ }^{1}$ Laboratory of Electrical and Industrial Systems, FEI, BP 32, University of Sciences and Technology Houari Boumediene, \\ Bab Ezzouar, Algiers, 16311 Algeria. \\ Phone number:+00213 554643321, e-mail: nacer3dz@gmail.com \\ ${ }^{2}$ Centre of Research and Development on Electricity and Gas, SONELGAZ, Ouled Fayet, Algeria \\ ${ }^{3}$ Electricity Transmission Power-Grid, SONELGAZ, 7 Rue Fernane Hanafi, Algiers, Algeria
}

\begin{abstract}
This work is devoted to an accurate investigation on the variations of the physicochemical properties of three transformer mineral oils of different levels of degradation submitted to moisture and electric discharges. The first one is a non treated new oil while the second is taken from a one year normal operating transformer and the third is extracted from a 15 years old operating transformer. The investigated parameters are breakdown voltage, relative permittivity $\varepsilon_{\mathrm{r}}$, dissipation factor (Tanס) and resistivity. Each oil peculiarities are determined through these parameters. Measurements on an on load operating transformer are reported to validate some laboratory obtained results.
\end{abstract}

\section{Key words}

Moisture, Transformer oil, Physicochemical properties, electric discharges.

\section{Introduction}

The electric properties of liquid insulators depend as much on the intrinsic parameters linked to their molecular constitution than on the extrinsic parameters related to their interaction with materials to which they are frequently associated, to the environment and conditions of exploitation of the transformer. The presence of water (moisture) dissolved in transformer oils is practically unavoidable.

It plays an ominous role for the dielectric strength as well in the oil [1] than in the paper [2] with which it is associated. This reduction is however of more importance for oil [3].

In any case, its quantity increases naturally with time [4], and if it comes to overtake a certain proportion, a deterioration process of the insulation system takes place, leading in most cases to the deterioration of the transformer if operations of oil treatment are not executed in time.

Generally, the contaminant elements and the more compromising with regard to the ageing of oil are water (moisture), particles and oxygen whose effect is sharply linked to the field of temperature to which the whole insulation is submitted.

Diagnose devices based on live measurements of moisture content are developed in order to activate an alarm if threshold quantities are reached as well in the oil than in the paper [5, 6].

This work is consecrated to the variations of dielectric strength, dissipation factor, resistivity and permittivity according to water content in three oils of different levels of deterioration. In the perspective of elementary diagnosis, we will try to clear some intrinsic and extrinsic elements linked to the presence of water and having an influence on oil properties.

\section{Experimental technique}

The three studied oils (Figure 1) are of naphtenic type and characterized since receipt; they represent in this state the check sample zero. Oil A is new and non-treated, and oils $\mathrm{B}$ and $\mathrm{C}$ are respectively new operating since one year and 15 years old operating. Their characteristics are presented on table I. The measurements are performed according to ISO 2909 standard for the viscosity $(\mu)$, ISO 2049 for the colour (Col), ISO 6618 for the acidity number (Ia), NFT 60-103 for the flash point (Pe) and Karl Fischer titration method for water content $\left(\mathrm{Wc}_{0}\right)$. The main purpose being to study the influence of water content on the different electric characteristics of the oil, we proceed in two stages: The first consists in doping the oil with distilled 
water in order to obtain samples of different water contents. The second concerns the measurement of the electric parameters (strength, $\tan \delta$, permittivity and resistivity).

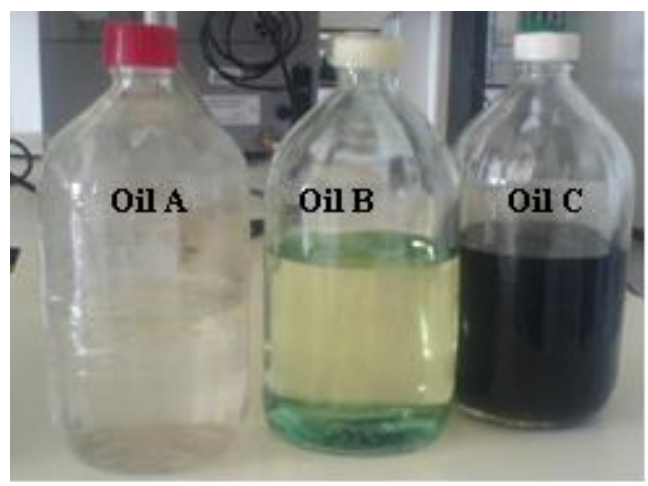

Fig. 1. Check samples of the three studied oils

Table I. Some physico-chemical properties of the three studied oils.

\begin{tabular}{|c|c|c|c|c|c|}
\hline $\begin{array}{c}\text { Type } \\
\text { of oil }\end{array}$ & $\begin{array}{c}\mathrm{Wc}_{0} \\
(\mathrm{ppm})\end{array}$ & $\begin{array}{c}\mu \\
\left(\mathrm{mm}^{2} / \mathrm{s}\right)\end{array}$ & Col & Ia & $\begin{array}{c}\text { Pe } \\
\left({ }^{\circ} \mathrm{C}\right)\end{array}$ \\
\hline $\mathrm{A}$ & 14.6 & 10.387 & $<0.5$ & 0.044 & 139 \\
\hline $\mathrm{B}$ & 9.5 & 10.962 & $<0.5$ & 0.039 & 150.5 \\
\hline $\mathrm{C}$ & 19.8 & 10.563 & $<0.5$ & 0.056 & 141 \\
\hline
\end{tabular}

For each oil, 9 samples are constituted, in addition to the check sample. They contain respectively an increasing number from 1 to 9 drops of distilled water. To well dissolve the water in oil, each sample was agitated and heated at constant speed $\left(800 \mathrm{~min}^{-1}\right)$ and temperature $\left(40^{\circ} \mathrm{C}\right.$ ), during 25 minutes. The measurements (water content, strength, $\tan \delta$, permittivity and resistivity) are executed without resting the oil to avoid water condensation and so measurement mistakes.

Measurements of breakdown voltage and other dielectric parameters of the three oils are executed in the same conditions. They are performed at ambient temperature and atmospheric pressure according to international standards [7].

The measurement of water content is performed directly after agitation, and in order to be closer to the most accurate value, at least three removals are done in different places of the bottle, because of the possible non homogeneity of the solution. The mean of the three measures is taken in consideration. For each test, the cell is rinsed three times or more, in order to keep the properties of the oil for which parameters are to be measured.

\section{Results and interpretations}

The executed tests yielded enough significant variations with regard to the dielectric strength. For the other studied parameters, the obtained variations are not as meaningful even though the doping of the oil has been increased up to important values overtaking $100 \mathrm{ppm}$.

During the executed tests, one met a certain number of difficulties that reverberated on the obtained results. Some are unavoidable because of the nature of the oil itself and the environment, due to the difficulty to reproduce in laboratory the exact conditions that exist inside the transformer. On the other hand, as oil and water are not compatible, it is not obvious to obtain a homogeneous mixture, from where the possible measurement errors of water content.

\section{A. Type of oil}

The appreciation of the initial condition depends on the results acquired from measurements of viscosity, colour and acidity number, flash point, water content, breakdown voltage, dissipation factor, resistivity and permittivity of the check sample. One notes that oil A is in good condition with regard to the values of previously defined characteristics. It presents however water content slightly elevated, due to the accumulation of air and humidity inside the barrel during the storage; that which is clearly shown by the value of acidity number which is $>0.03$. Oil $\mathrm{B}$ is also in good condition, because it has been treated before its set in service.

Oil $\mathrm{C}$ begins to be aged, what proves the elevated values of the acidity number, water content and dissipation factor which are greater than standard recommendations, as well as the weak values of the strength and resistivity. It is observable through the abnormal coloration of this oil visible to the naked eye, due to the presence of sediments and particles.

\section{B. Breakdown voltage}

Breakdown voltage is the basic element to judge the condition of mineral oil. Figure 2 represents the variation of breakdown voltage as a function of water content (Wc). For each value of measured water content in ppm correspond a mean value of breakdown voltage that is obtained after 6 successive discharges consistently to IEC 156 recommendations.

For the three liquids, the strength represents the most explicit characteristic as for the influence of water contents on the dielectric quality of the oil, whatever its quality of origin: new treated, not treated or used. It decreases in a sharp manner, surrendering the oil non conform after having overtaken about ten ppm of water in the oil. This limit is in agreement with standards and manufacture characteristics. Moisture degrades in the same way the oil whatever its situation of origin. Breakdown voltage decreases to about $25 \mathrm{kV}$ for an oil containing $50 \mathrm{ppm}$ of water and to $10 \mathrm{kV}$ when it contains $100 \mathrm{ppm}$.

The strength at point 0 for the two first oils $A$ and $B$ is almost the same; it is located around an average voltage of $65 \mathrm{kV}$. It means that the studied oil presents a good dielectric strength until a concentration of $15 \mathrm{ppm}$ of water clearly greater than the $10 \mathrm{ppm}$ set by specific standards. On the other hand, Oil C presents a bad strength $(45 \mathrm{kV}$ at point 0$)$ in spite of the modest relative value of its water content, what suggests that this latter is not the only decisive parameter as for the strength of the oil. The dielectric strength decreases with water content in an almost independent manner with regard to the initial condition of the oil. 


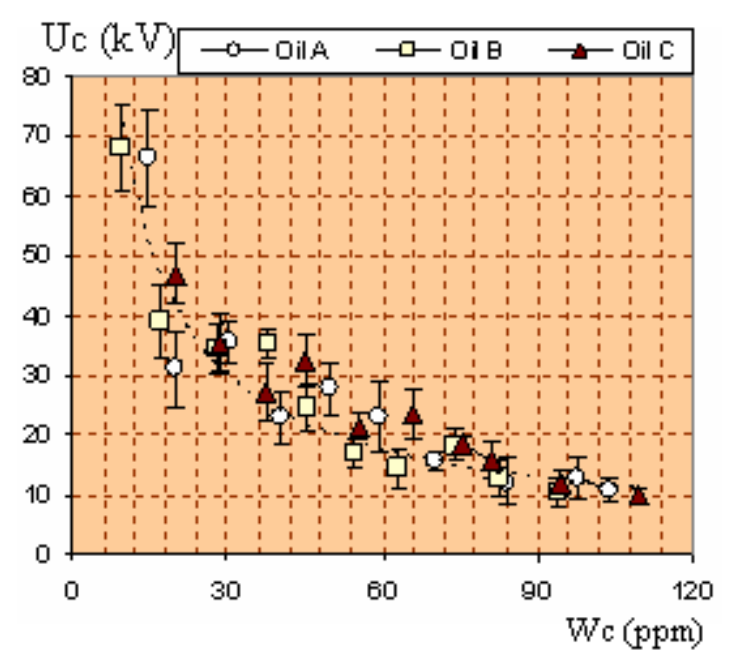

Fig. 2. Variations of breakdown voltage Uc as a function of water content Wc for the three oils A, B and C

\section{Dissipation factor (Tan $\delta)$}

The variation of the dissipation factor is one of the elements that inform us about the state of the mineral oil. The measurements performed about the variations of $\tan \delta$ while water content in the oil is varied are presented in Figure 3. The values of $\tan \delta$ are different from an oil to the other. We measured values varying between 0.5 and $2.10^{-3}$ for oil $\mathrm{A}$, between 1,5 and $3,5 \cdot 10^{-3}$ for oil $\mathrm{B}$ and between 5 and $9,5 \cdot 10^{-3}$ for oil $\mathrm{C}$.

For given oil, the dissipation factor is one of the factors that present the less sensitivity to water content variations. There is however a certain sensitivity for the used oil whose variations are relatively difficult to interpret. This factor expresses the relation between the resistive and capacitive natures of the oil. As the dissolution of water in oil affects the two characters simultaneously, the interpretation of the obtained results becomes difficult. It is thus preferable to study these two characters separately through the measurement of the resistivity and relative permittivity variations.

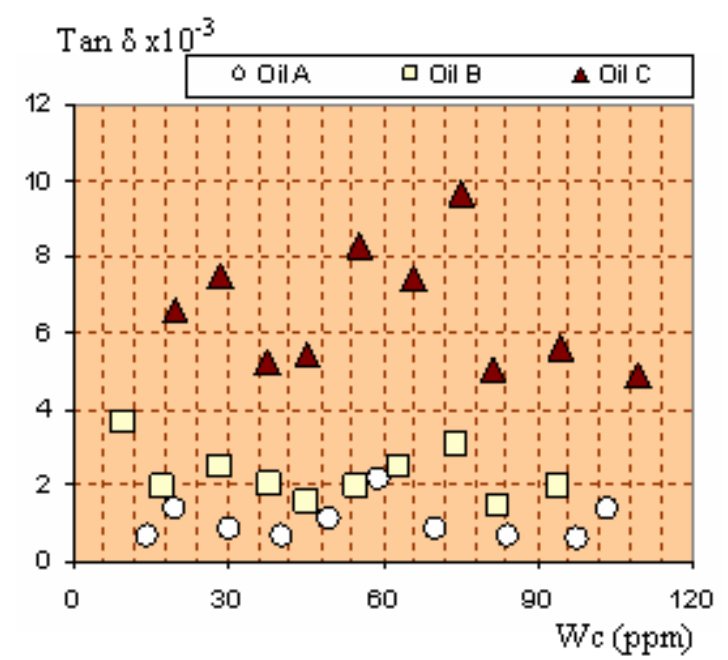

Fig. 3. Influence of water content on the dissipation factor $(\tan \delta)$ for the three oils.

\section{Permittivity $\left(\varepsilon_{\mathrm{r}}\right)$}

The relative permittivity is a non dimensional value; it depends on the internal structure of the oil and characterizes the polarity between molecules. Figure 4 presents the variations of the permittivity $\varepsilon_{\mathrm{r}}$ of the oil while its water content is varied. It is also one of the principal characteristics of the oil the most difficult to impair. Despite the advanced deterioration of oil C, its permittivity is less different from the one of new oil (oil B). It remains likewise unaltered with water contents increasing until 100 ppm. However, it is well known [8] that permittivity can point out a certain sensitivity while studying its two intrinsic components separately $\left(\varepsilon_{\mathrm{r}}=\varepsilon_{\mathrm{r}}-\right.$ j. $\varepsilon_{\mathrm{r}}$ ) by performing measurements in a large frequency domain.

These results mean that the addition of quantities of water to oil doesn't act on its own microscopic properties; it rather acts on its macroscopic properties. The relatively small values of permittivity measured in oil A could be due to the presence of particles of water of very small dimension or solid particles in the non treated oil.

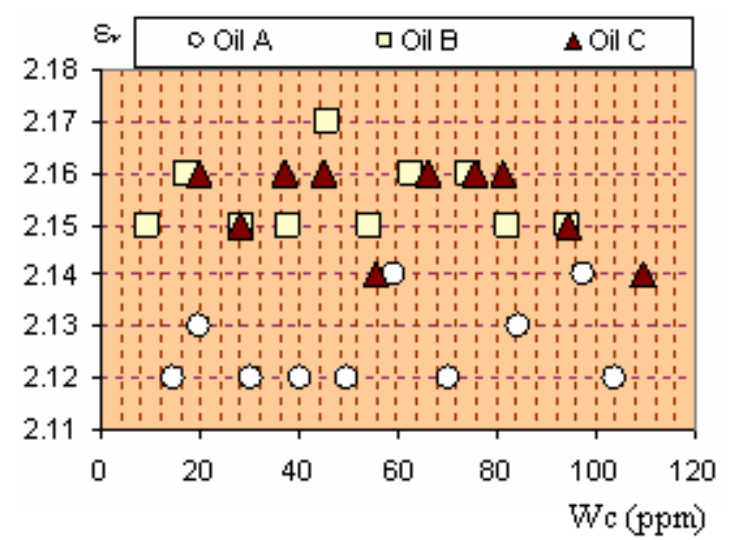

Fig. 4. Relative permittivity variations as a function of water content for the three oils.

\section{E. Resistivity $(\rho)$}

Resistivity expresses the resistive character of oil. It depends on the molecular aspect and existing particles in the liquid. Figure 5 represents the variations of the mean value of the resistivity $\rho$ as a function of water content in the three studied oils. The measured values differ from an oil to the other. Oil A presents a mean value of $350.10^{9}$ $\Omega . \mathrm{m}$. The one of oil B is located around $150.10^{9} \Omega . \mathrm{m}$ whereas oil C presents an average value of $70.10^{9} \Omega . \mathrm{m}$. For given oil, the resistivity with regard to water content presents the most unexpected variations because it presents globally a tendency to increase with moisture. This tendency is marked by the transition by a minimum point. These results denote the bad miscibility of distilled water in naphtenic mineral oil, in spite of the long phases of agitation applied to the mixture.

Since measurements of resistivity necessitate a relatively elevated period, particles of water will have sufficiently time to be decanted and to go down towards the bottom, tending to give again to the sample its initial properties, i.e. those of the check sample. The minimum of resistivity 
evidenced for each type of oil could correspond to a zone of water miscibility in oil. It is of the order of $20 \mathrm{ppm}$ for oil A, 25 ppm for oil B and 40 ppm for oil C.

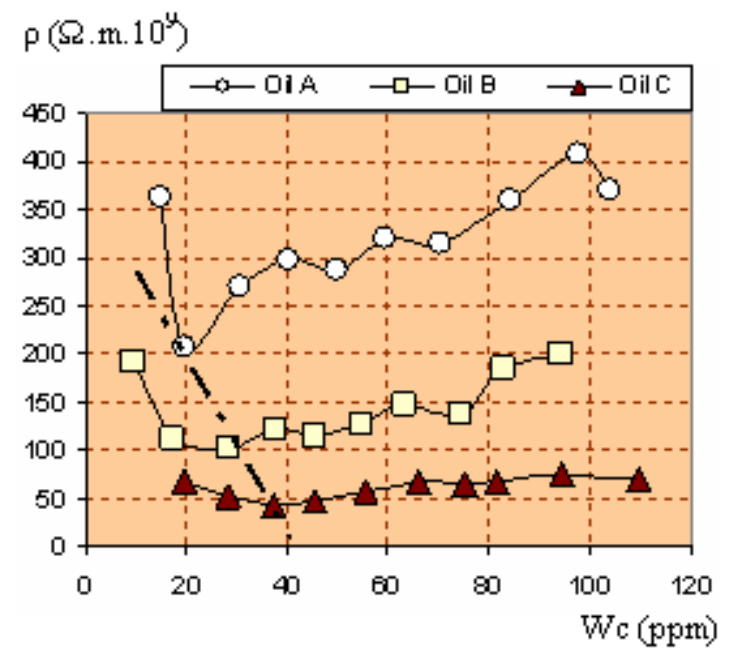

Fig. 5. Variations of resistivity as a function of water content for the three oils.

\section{Influence of discharges of low energy on water content}

Partial discharge currents are ranged from few to hundreds of $\mathrm{mA}$ [9]. They represent the principal element leading to a fast degradation of the insulation system [10]. In order to appreciate their influence on water content and the variations of certain properties of the oil containing abnormal quantity of water, we submitted a sample of new non treated oil having an initial water content of $32.5 \mathrm{ppm}$ to series of discharges of weak energy level (about $20 \mathrm{~mJ}$ ). Figure 6 expresses the variations of water content according to the number of discharges occurred in the studied oil. It presents a tendency to the reduction with discharges. It means that the applied discharges provoke a vaporisation of water traces that are present in the zone of discharge occurrence.

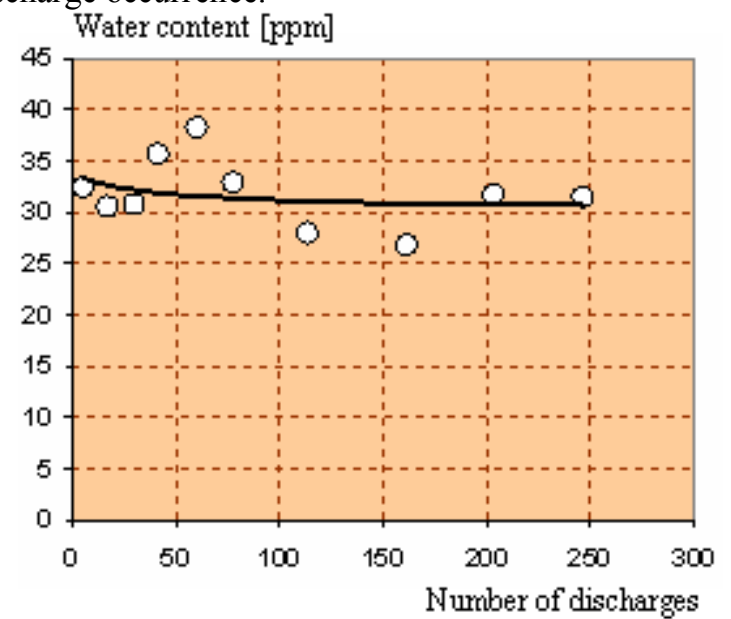

Fig. 6. Water content (in ppm) as a function of the number of discharges.

The taken measurements present however a fluctuated behaviour due to the fact that only a small part of liquid is drown for measurement; knowing that after a series of discharges, inhomogeneous zones are generated in the whole of the liquid, in spite of the agitation imposed to the liquid during all the tests. The different approximations: linear, logarithmic, exponential, power and mobile average set in evidence this trend to the reduction of moisture as the number of discharges increases.

It is possible to observe a reduction of about $3 \mathrm{ppm}$ after 250 discharges. The tendency to decrease is enough weak to hope for an amelioration of water content of the oil by its submission to discharges of weak energy levels. If the relation is supposed linear, it will be necessary 1750 discharges of energy level of those that we applied to reduce water content to the value of $10 \mathrm{ppm}$, given by standards as a satisfactory limit. Moreover, as weak are they, these discharges affect negatively and with enough significant manner other parameters of oil, such as the resistivity and the dissipation factor (Figures 7 and 8). In proportion as the liquid is submitted to discharges, the timid decrease of water content is accompanied by a significant increase of $\tan \delta$ and a reduction of $\rho$. On the other hand, its relative permittivity remains unaltered.

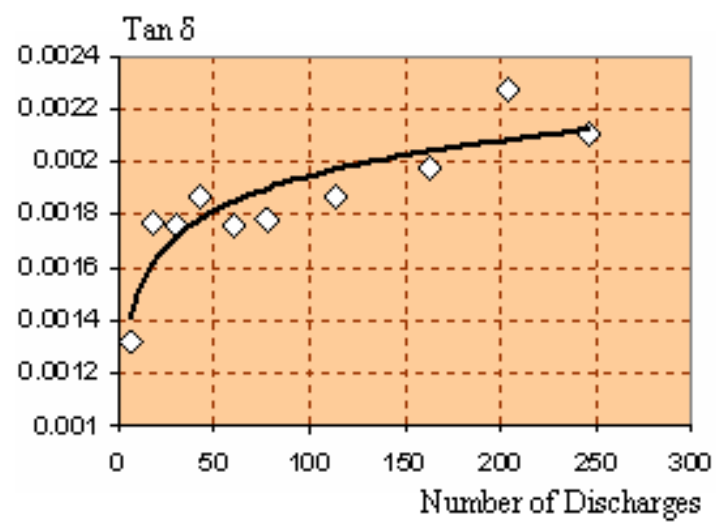

Fig. 7. Variations of dissipation factor $(\operatorname{Tan} \delta)$ as a function of the number of discharges.

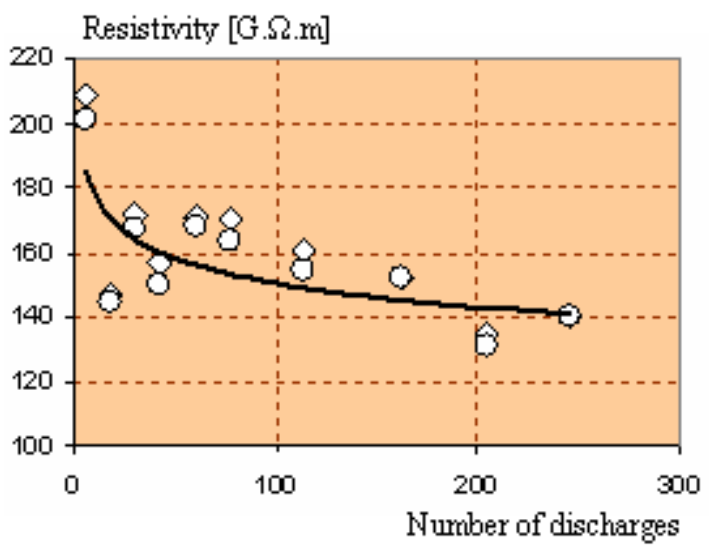

Fig. 8. Max and min Resistivity $\rho$ (G. $\Omega . m$ ) as a function of the number of discharges.

\section{Real case of an operating transformer}

In order to appreciate, in a real situation, the influence of water content on the properties of the oil, especially its breakdown voltage, we reported on Figure 9 these variations for the same type of oil taken at definite intervals of time from an operating transformer $(60 / 30 \mathrm{kV}$; 25 MVA; cooling: ONAN-ONAF). This transformer was in partial default undergone treatments after each oil 
removal if it proves out to be necessary. In order to quantify the difference between these variations and the mean values of those obtained on the same type of oil but doped in laboratory, we present them on the same Figure.

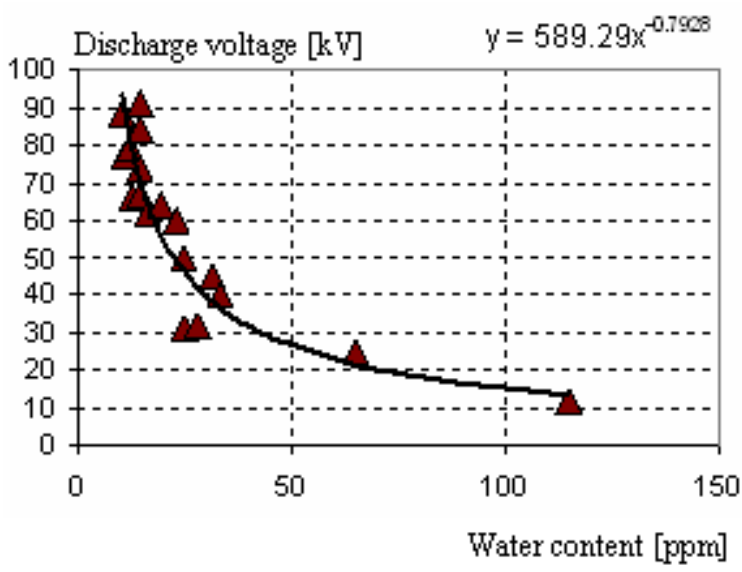

Fig. 9. Breakdown voltage as a function of water content for an oil of the same type but taken at different intervals of time from an operating transformer.

It appears that the dielectric strength of oil is reduced severely by the increase of water content. It varies according to a function of type: $\mathrm{Uc}=590 . \mathrm{Wc}^{-0.8}$, where $U c$ is the breakdown voltage and $W c$ the water content.

Takings and treatments of the oil have been carried out between June, 13-2006 and September, 23-2006 with intervals of time varying between 2 and 20 days according to the result of the measurements. Figure 10 shows the variations of breakdown voltage and water content according to the chronology of removals and treatments, from the first to the $110^{\text {th }}$ day. It is necessary to mention that some takings and treatments have been executed after switching out the transformer. This stopping has been imposed by the difficulty to bring back the oil at acceptable strength levels. It is then necessary to discuss the conditions of the transformer switching out and the chosen intervals between operations of taking/treatment that were relatively long.

It is true that intervals between takings foreseen by IEEE Std C157.104 are yearly, quarterly, monthly, weekly or daily when the diagnosis evidences an advanced deterioration of the insulation. This diagnosis is based on the quantities of dissolved gas present in oil. For the present case of diagnosis on water content in the studied oil, the adopted chronology and measured moisture in the paper are presented as histograms in Figure 11. This choice urged the operators in charge of the transformer maintenance to adopt a long chronology of 110 days having moreover constrained its stopping during 29 days.

For this effect, we propose on Figure 11, by continuous curve, a chronology of treatments that would have must be applied in order to bring into use again the transformer on acceptable conditions (breakdown voltage $>50 \mathrm{kV}$, water content $<15 \mathrm{ppm}$ and moisture content in paper $<2$ $\%)$. The period of the treatments would be reduced to only 55 days.

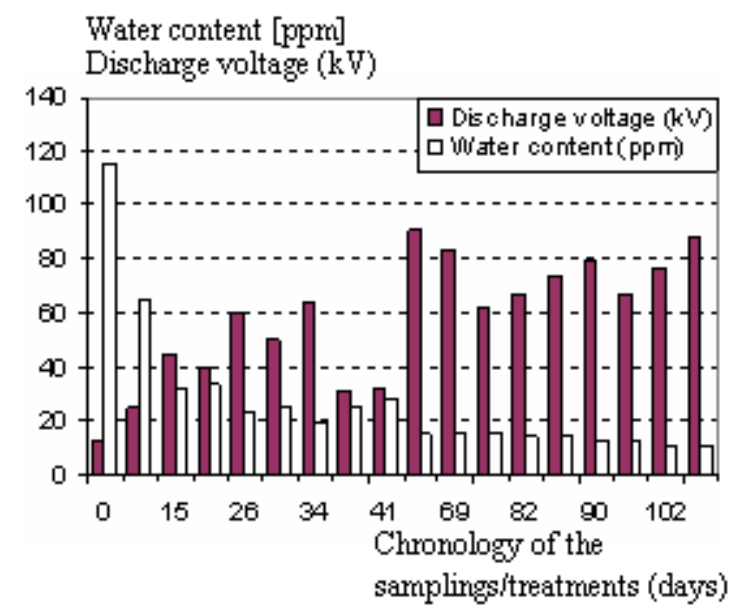

Fig.10. Breakdown voltage and water content as a function of the delay time between samplings and eventual treatment.

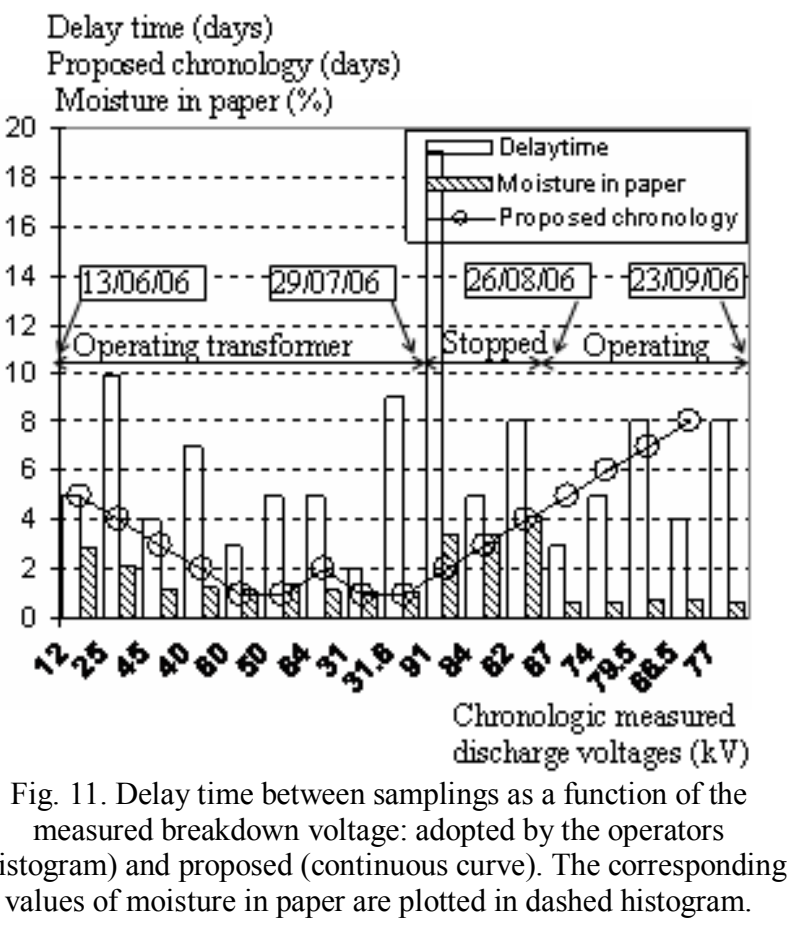

\section{Conclusion}

Water content in a dissolved form in oil is an essential parameter that affects the dielectric strength (breakdown voltage). It is not however the single decisive parameter. Its action is combined with those of other parameters. The ageing of oil through which reactions of acidification triggered by the presence of water acts so that weak quantities of water permit to reduce appreciably the insulating properties of this oil.

This is illustrated by the measured values of $\tan \delta$ and resistivity of oil A which are of better quality that those of oil $\mathrm{B}$, whereas this latter is sensed to have been treated. Through the different tests that we achieved and the observed events during maintenance and surveillance of operating transformers, it appears in a technical point of view that the progressive deterioration of the oil is due to the simultaneous combination of physical, chemical and electric stresses. The increase of moisture plays a major role in the acceleration of the process of this deterioration. The consequences can be expressed by an increase of the 
losses and acidity at long-term, and decrease on resistivity when the oil and water are in the phase of miscibility and decrease of the dielectric strength whatever the initial type of oil.

\section{References}

[1] I. Fofana, H. Borsi and E. Gockenbach 'Fundamental investigations on some transformer liquids under various outdoor conditions", IEEE Trans. on Diel. and Elect. Ins. Vol.8 No.6 Décember 2001

[2] C. Ekanayake, S.M. Gubanski, A. Graczkowski and K. Walczak, 'Frequency response of oil impregnated pressboard and paper samples for estimating moisture in transformer insulation', IEEE Trans on Power Delivery, Vol.21 No.3, July 2006.

[3] P. M. Balma, R. C. Degeneff, H.R.Moore and L.B. Wagenaar, "The effects of long term operation and system conditions on the dielectric capability and insulation coordination of large power transformers", IEEE Trans on Power Delivery, Vol.14 No.3, July 1999.

[4] M. A. Abdel Ouahab, M.M. Hamada, A.G. Zeitoun and G. Ismail, "A newly modified forced oil cooling system and its impact on in-service transformer oil characteristic', IEEE Trans on Power Del., Vol.18 No.3, July 2003.
[5] B. Garcia, J.C. Burgos, A.M Alonso and J. Sanz, 'A moisture-in-oil model for power transformer monitoring-Part I : Theoretical foundation', IEEE Trans on Power Delivery, Vol.20 No.2, April 2005, pp. 1417-1422.

[6] B. Garcia, J.C. Burgos, A.M Alonso and J. Sanz, 'A moisture-in-oil model for power transformer monitoring-Part II: Experimental verification', IEEE Trans on Power Delivery, Vol.20 No.2, April 2005, pp. 1423-1429.

[7] H. Moulai, I. Khelfane, A. Nacer and A. Debche, "Water content influence on physico-chemical properties of a transformer naphtenic oil',, Proc. Int. Conf. on Power and Energy Systems (Corfu, Greece) (Acta Press), 23-25 June 2008, pp. 249-253

[8] C.T. Dervos, C.D.Paraskevas, P. Skafidas and P. Vassiliou, 'Dielectric characterisation of power transformer oils as a diagnostic life prediction method", IEEE Electrical Insulation Magazine, Vol. 21, No. 1, January/February 2005.

[9] A. Beroual and H. Moulai, 'Prebreakdown currents analysis in insulating oils under AC Voltages - Relation to breakdown', Archives of Electrical Engineering (AEE) Vol. L No.1, 2001, pp.111-124

[10] H. Moulai, A. Nacer and A. Beroual, 'Characterisation of partial discharges in mineral oil', Proc. Int. Conf. on Power and Energy systems (Rhodes, Greece) (Acta Press), 26-28 June 2006, No 468-140. 